\title{
Impact of HLA class I high-resolution mismatches on chronic graft-versus-host disease and survival of patients given hematopoietic stem cell grafts from unrelated donors
}

\author{
HT Greinix ${ }^{1}$, I Faé2, B Schneider ${ }^{3}$, A Rosenmayr ${ }^{2,4}$, A Mitterschiffthaler ${ }^{4}$, B Pelzmann ${ }^{4}$, P Kalhs ${ }^{1}$, \\ K Lechner ${ }^{1}$, WR Mayr ${ }^{2}$ and GF Fischer ${ }^{2}$ \\ ${ }^{1}$ Department of Medicine I, Bone Marrow Transplantation, Medical University of Vienna, Austria; ${ }^{2}$ Department of Blood Group \\ Serology, Medical University of Vienna, Austria; ${ }^{3}$ Institute of Medical Statistics and Documentation, University of Vienna, Austria; \\ and ${ }^{4}$ Austrian Bone Marrow Donor Registry, Vienna, Austria
}

\section{Summary:}

There is consensus that matching of unrelated donors (URD) and patients for HLA class II alleles improves the outcome of hematopoietic stem cell transplantation (HSCT). However, the significance of HLA class I allelic mismatches for transplant outcome is under discussion and reports on long-term effects like chronic graft-versus-host disease (GVHD) are rare. Thus, we investigated the association of human leukocyte antigen (HLA) class I allele mismatches and outcome in 144 patients given HSCT from URD who were matched for HLA-DRB1, DRB3/4/5, and DQB1 alleles. The risk of chronic GVHD was significantly increased in patients with class I mismatched donors, the mismatch either detected by low- or high-resolution typing. A single HLA class I allele mismatch significantly increased the risk of chronic GVHD in multivariate analysis. Overall survival was significantly reduced in patient/donor pairs with more than one-allele class I mismatch. Thus, selection of unrelated donors for transplantation should be based on high-resolution HLA class I typing.

Bone Marrow Transplantation (2005) 35, 57-62.

doi:10.1038/sj.bmt.1704741

Published online 8 November 2004

Keywords: HLA class I; high resolution typing; chronic GVHD

Allogeneic hematopoietic stem cell transplantation (HSCT) is a well-established curative therapy for lymphohematopoietic and congenital metabolic disorders. ${ }^{1,2}$ Since a genotypically matched sibling donor is available for only $20-30 \%$ of the patients in need of a transplant, an increasing number of HSCT from human leukocyte antigen

Correspondence: Dr HT Greinix, AKH Wien, Klinik fuer Innere I, Knochenmarktransplantation, Waehringer Guertel 18-20, A-1090

Vienna, Austria; E-mail: hildegard.greinix@meduniwien.ac.at

Received 16 July 2004; accepted 17 September 2004

Published online 8 November 2004
(HLA)-matched unrelated donors (URD) has been performed during the last years. ${ }^{3,4}$ URD transplants are usually associated with a higher incidence of posttransplant complications compared to HLA-genoidentical transplants. ${ }^{3,5-10}$ This is mainly due to unresolved HLA incompatibilities or to mismatched loci not tested for by conventional tissue typing. The use of molecular typing techniques for all HLA loci has demonstrated that serological matching is insufficient to ensure an allelic match. ${ }^{8}$ High-resolution DNA-based typing for both HLA class I and class II alleles has greatly improved accuracy of donor selection resulting in improved outcome of HSCT in HLA-identical donor/patient pairs. ${ }^{6-9,11-16}$

Controversies remain as to whether mismatches which can only be detected using high-resolution (allele level) nucleic acid techniques are more permissive of clinical success than those mismatches which can be detected using serology or comparable low-resolution (antigen level) typing approaches.

Especially, reports on the impact of HLA allelic disparities on incidence and severity of chronic graftversus-host disease (GVHD) are rare. ${ }^{17,18}$ Here, we report a single center study with long follow-up on 144 patients given HSCT from URD all matched for DRB1, DRB3, DRB4, DRB5, and DQB1 alleles typed at the highresolution level. We show that HLA class I allelic mismatches significantly increase the risk of chronic GVHD and thus, should be taken into account when searching for an unrelated donor.

\section{Patients and methods}

\section{Patients}

We performed a retrospective analysis of 144 consecutive patients transplanted from URD at the Medical University of Vienna from September 1994 until July 2003. Pretransplant characteristics of the patients are shown in Table 1 . All except three patients were conditioned for transplantation with cyclophosphamide and fractionated total body irradiation. For GVHD prophylaxis, all except five received cyclosporine A (CSA) and methotrexate. A total of 100 
Table 1 Characteristics of donors and patients

\begin{tabular}{|c|c|c|c|c|}
\hline & All & $\begin{array}{l}\text { Allelic } \\
\text { match }\end{array}$ & $\begin{array}{c}\text { Allelic } \\
\text { mismatch }\end{array}$ & $\begin{array}{c}\text { Antigen } \\
\text { mismatch }\end{array}$ \\
\hline No. of patients & 144 & 82 & 23 & 39 \\
\hline Median age of patients (years) & 37 & 38 & 36 & 35 \\
\hline Range & $18-55$ & $18-55$ & $22-54$ & $21-50$ \\
\hline Median age of donors (years) & 37 & 36 & 38 & 37 \\
\hline Range & $18-53$ & $18-52$ & $24-49$ & $20-53$ \\
\hline \multicolumn{5}{|l|}{ Gender (donor/patient) } \\
\hline $\mathrm{M} / \mathrm{M}$ & 67 & 36 & 13 & 18 \\
\hline $\mathrm{M} / \mathrm{F}$ & 28 & 16 & 4 & 8 \\
\hline$F / F$ & 33 & 20 & 4 & 9 \\
\hline $\mathrm{F} / \mathrm{M}$ & 16 & 10 & 2 & 4 \\
\hline Donor pregnancy ${ }^{\mathrm{a}}$ & 30 & 24 & 1 & 5 \\
\hline \multicolumn{5}{|l|}{ Diagnosis } \\
\hline CML & 53 & 31 & 10 & 12 \\
\hline AML & 40 & 20 & 7 & 13 \\
\hline $\mathrm{ALL}+\mathrm{AL}$ & 31 & 18 & 3 & 10 \\
\hline SAA & 2 & 2 & 0 & 0 \\
\hline NHL & 7 & 6 & 1 & 0 \\
\hline MDS & 10 & 5 & 2 & 3 \\
\hline MM & 1 & 0 & 0 & 1 \\
\hline \multicolumn{5}{|l|}{ Disease risk } \\
\hline Standard ${ }^{\mathrm{b}}$ & 80 & 44 & 13 & 23 \\
\hline High & 64 & 38 & 10 & 16 \\
\hline \multicolumn{5}{|l|}{$C M V(\text { donor } / \text { patient })^{\mathrm{c}}$} \\
\hline Pos/Pos & 26 & 20 & 4 & 2 \\
\hline $\mathrm{Pos} / \mathrm{Neg}$ & 27 & 14 & 5 & 8 \\
\hline $\mathrm{Neg} / \mathrm{Neg}$ & 44 & 24 & 7 & 13 \\
\hline $\mathrm{Neg} / \mathrm{Pos}$ & 42 & 22 & 5 & 15 \\
\hline $\mathrm{ABO}$ incompatibility ${ }^{\mathrm{d}}$ & 87 & 47 & 13 & 27 \\
\hline Rhesus incompatibility ${ }^{d}$ & 46 & 32 & 5 & 9 \\
\hline
\end{tabular}

No=number; $\mathrm{M}=$ male; $\mathrm{F}=$ female; $\mathrm{CML}=$ chronic myelogenous leukemia; $\mathrm{AML}=$ acute myelogenous leukemia; $\mathrm{ALL}=$ acute lymphoid leukemia; $\mathrm{AL}=$ acute biphenotypic leukemia; $\mathrm{SAA}=$ severe aplastic anemia; $\mathrm{NHL}=$ non-Hodgkin's lymphoma; $\mathrm{MDS}=$ myelodysplastic syndrome; $\mathrm{MM}=$ multiple myeloma; $\mathrm{CMV}=$ cytomegalovirus; pos = positive; neg = negative.

${ }^{\mathrm{a}}$ Four results missing.

${ }^{\mathrm{b} C}$ Complete remission and chronic phase.

${ }^{\mathrm{c}}$ Five results missing.

${ }^{\mathrm{d}}$ One result missing.

patients were given unmanipulated bone marrow (BM) and 44 unmanipulated peripheral blood stem cells (PBSC). The clinical diagnosis of GVHD was confirmed by histopathology and graded as published. ${ }^{19,20}$

The protocols used had been approved by the ethical committee of the University of Vienna. Informed consent was obtained from all patients.

\section{HLA typing and strategy for the selection of donors}

Low-resolution typing for HLA-A, B, C, and DR was performed with a standard two-stage complement-dependent test of microtoxicity ${ }^{21}$ or by ligation-based typing. ${ }^{22}$ For high-resolution typing, sequencing analyses of exons 13 of HLA class I alleles and exons 2 of HLA class II alleles were performed. ${ }^{23}$

We aimed at comprehensive matching of HLA-DRB1, DRB3 $/ 4 / 5$, and DQB1 alleles as assessed by high-resolution typing and matching of HLA-A, B, or C antigens as assessed by low-resolution typing. High-resolution typing, of HLA class I alleles was performed prospectively.

\section{Statistical analysis}

Data were analyzed as of February 1, 2004. Patients were considered evaluable for engraftment if they survived 21 days after transplant and evaluable for chronic GVHD if they survived at least 80 days. Overall survival (OS) and disease-free survival (DFS) were estimated using the Kaplan-Meier method, whereas relapse, transplant-related mortality (TRM), acute and chronic GVHD were calculated as cumulative incidence ${ }^{24}$ to adjust the analysis for competing risks. For the analysis on acute and chronic GVHD, both relapse and death in remission in the absence of GVHD were considered competing events; for the analysis on relapse incidence, death was the competing event, whereas relapse was the competing event for the analysis on TRM. Comparisons of cumulative incidences between different groups were made by k-sample Gray 
Table 2 Number and types of HLA class I mismatches observed in 144 donor/patient pairs matched for HLA-DRB1, DRB3/4/5, and DQB1

\begin{tabular}{|c|c|c|c|c|c|c|}
\hline \multirow[t]{2}{*}{$\begin{array}{l}\text { Mismatches by locus } \\
\text { Locus }\end{array}$} & \multicolumn{2}{|c|}{ Low resolution } & \multicolumn{2}{|c|}{ High resolution } & & \\
\hline & Match & Mismatch & Match & Mismatch & & \\
\hline HLA-A & 132 & 12 & 125 & 19 & & \\
\hline HLA-B & 139 & 5 & 123 & 21 & & \\
\hline HLA-C & 122 & 22 & 110 & 34 & & \\
\hline \multicolumn{7}{|c|}{ Details of HLA class I allelic mismatching in $H L A-D R B 1, D R B 3 / 4 / 5$, and $D Q B 1$ matched pairs } \\
\hline & A & $B$ & $C$ & $A$ and $B$ & $A$ and $C$ & $B$ and $C$ \\
\hline One mismatch & 14 & 10 & 22 & & & \\
\hline Two mismatches & 1 & & 3 & 3 & 1 & 6 \\
\hline Three mismatches & & & & & & 1 \\
\hline Four mismatches & & & & & & 1 \\
\hline
\end{tabular}

test. $^{24}$ Log-rank test statistics were used to evaluate the univariate effects of HLA class I antigen and allele incompatibility on outcome. For HLA-A, B, and C, donor/recipient matching was considered in three categories: high-resolution match, low-resolution match/highresolution mismatch, and low-resolution mismatch.

Additionally, other factors, namely patient and donor age, patient and donor sex, diagnosis, disease status at HSCT, and stem cell source were tested in univariate analysis. Multivariate analyses were carried out by Cox proportional hazards regression modelling of OS and relapse. The median observation time of surviving patients is 45 (range, 6-114) months.

\section{Results}

\section{Degree of HLA class I disparity}

Number and types of HLA class I mismatches observed are shown in Table 2. In total, 105 donor/patient pairs $(73 \%)$ had no HLA class I antigen level mismatches, whereas 39 were mismatched for one antigen (12 for HLA-A, five for HLA-B, and 22 for HLA-C). High-resolution typing reduced the number of HLA class I identical donor/patient pairs to $82(57 \%)$. A single class I allelic mismatch was present in $46(32 \%)$, more than one mismatch was observed in $16(11 \%)$ donor/patient pairs. Overall, high-resolution typing revealed HLA class I mismatches not detected by low-resolution typing in $35(24 \%)$ donor/patient pairs.

\section{GVHD}

Stable engraftment occurred in all patients. The cumulative incidence of acute GVHD was $66 \% \quad(95 \%$ confidence interval (CI), 59-74\%) for the whole cohort and was $67 \%$ (95\% CI, 58-78\%), 57\% (95\% CI, 39-81\%), and 69\% $(95 \%$ CI, 56-85\%) in the high-resolution match, lowresolution match/high-resolution mismatch and low-resolution mismatch, group and thus, not significantly different. The corresponding percentages for acute GVHD grades III-IV were with 22,18 , and $22 \%$, also not significantly $(P=0.45)$ different.

The cumulative incidence of chronic GVHD at 3 years was $32 \%(95 \% \mathrm{CI}, 25-40 \%)$ for the whole group. It was

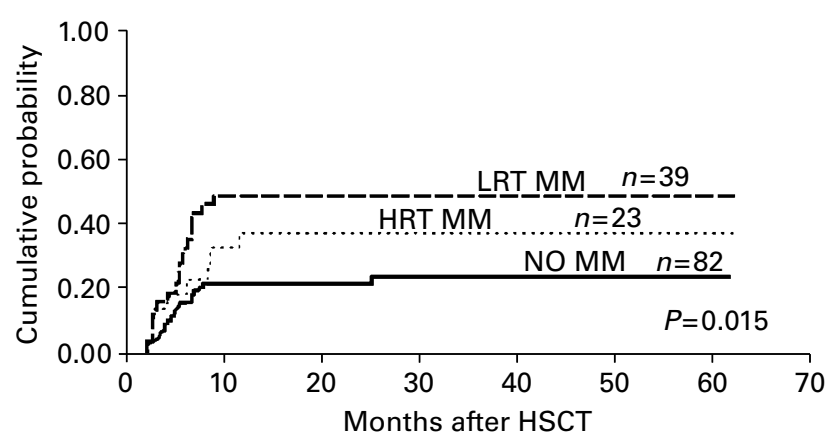

Figure 1 HLA class I disparities and incidence of chronic GVHD. Cumulative incidence of chronic GVHD at 3 years is shown according to the degree of HLA class I disparity between donor and recipient. Groups comprise patients with high-resolution match (thick solid line; HLA-A, B, and $\mathrm{C}$ antigen and allele match), low-resolution match/high-resolution mismatch (fine, broken line; antigen match but allele mismatch of HLA-A, $\mathrm{B}$ or $\mathrm{C}$ ) and low-resolution mismatch (thick broken line; HLA-A, B, or C antigen mismatch) donors.

$23 \%(95 \%$ CI, $15-34 \%), 37 \%$ (95\% CI, 21-64\%), and $48 \%(95 \%$ CI, 35-67\%) for patients with high-resolution match, low-resolution match/high-resolution mismatch, and low-resolution mismatch donors. (Figure 1, $P=0.015)$. The corresponding percentages for chronic extensive GVHD were 12, 13 , and $23 \%$ and not significantly $(P=0.08)$ different. A single HLA class I allele mismatch significantly increased the risk of chronic GVHD at 3 years from $21 \%(95 \% \mathrm{CI}, 14-32 \%)$ to $44 \%(95 \% \mathrm{CI}$, $33-58 \%)$. In both univariate and multivariate analysis (Table 3), only HLA class I disparity significantly increased the risk of chronic GVHD $(P=0.0007)$.

\section{Outcome}

A total of 24 patients died within 60 days after HSCT of infections $(n=6)$, GVHD and infections $(n=3)$, GVHD and multiorgan failure (MOF, $n=3), \operatorname{MOF}(n=6)$, venoocclusive disease $(n=3)$, bleeding $(n=1)$, and relapse $(n=2)$. The cumulative incidence of TRM at 3 years was $24 \%(95 \%$ CI, 16-35\%), 26\% (95\% CI, 13-52\%), and $34 \%(95 \%$ CI, 21-52\%) for patients without HLA class I allelic disparities, low-resolution match/high-resolution 
Table 3 Multivariate analysis of HLA compatibility/transplant outcome

\begin{tabular}{|c|c|c|c|c|c|}
\hline & $\begin{array}{c}O S \\
P\end{array}$ & $\begin{array}{c}D F S \\
P\end{array}$ & $\begin{array}{c}a G V H D \\
P\end{array}$ & $\begin{array}{c}c G V H D \\
P\end{array}$ & $\begin{array}{c}R e l \\
P\end{array}$ \\
\hline \multicolumn{6}{|l|}{ HLA compatibility } \\
\hline HRT-M/HRT-MM/LRT MM & 0.27 & 0.46 & 0.62 & 0.0007 & 0.49 \\
\hline \multicolumn{6}{|l|}{ Disease stage } \\
\hline Standard/high risk & 0.01 & 0.0003 & 0.19 & 0.29 & $<0.0001$ \\
\hline \multicolumn{6}{|l|}{ Stem cell source } \\
\hline $\mathrm{BM} / \mathrm{PBSC}$ & 0.39 & 0.62 & 0.73 & 0.36 & 0.15 \\
\hline \multicolumn{6}{|l|}{ Patient age } \\
\hline$<40 / \geqslant 40$ years & 0.37 & 0.68 & 0.53 & 0.12 & 0.78 \\
\hline \multicolumn{6}{|l|}{ Donor age } \\
\hline$<40 / \geqslant 40$ years & 0.74 & 0.33 & 0.72 & 0.44 & 0.42 \\
\hline \multicolumn{6}{|l|}{ Patient $C M V$ status } \\
\hline Positive/negative & 0.69 & 0.58 & 0.79 & 0.74 & 0.55 \\
\hline \multicolumn{6}{|l|}{ Donor $C M V$ status } \\
\hline Positive/negative & 0.75 & 0.74 & 0.55 & 0.85 & 0.72 \\
\hline
\end{tabular}

OS = overall survival; DFS = disease-free survival; aGVHD = acute graft-versus-host disease; cGVHD = chronic graft-versus-host disease; rel = relapse; HRT-M = high-resolution typing match; HRT-MM = high-resolution typing mismatch; LRT $M M=$ low-resolution typing mismatch; $\mathrm{BM}=$ bone marrow; $\mathrm{PBSC}=$ peripheral blood stem cells; $\mathrm{CMV}=$ cytomegalovirus.

mismatch, and low-resolution mismatch donors and thus, not significantly different.

At the end of study, 42 patients $(31 \%)$ had experienced relapse including $14(17.5 \%)$ with standard risk and 28 (44\%) with high-risk disease prior to HSCT. The cumulative incidence of relapse at 3 years was $35 \%(95 \% \mathrm{CI}$, $26-48 \%$ ), $27 \%$ (95\% CI, $14-53 \%$ ), and $27 \%$ (95\% CI, $16-$ $46 \%$ ) for patients with high-resolution match, low-resolution match/high-resolution mismatch and low-resolution mismatch, donors and not significantly different. Only disease status at transplant $(P \leqslant 0.0001)$ had a significant impact on relapse as shown in Table 3.

The Kaplan-Meier estimates for OS at 3 years were $49 \%$ (95\% CI, 6-51\%), 50\% (95\% CI, $11-50 \%)$, and 38\% (95\% CI, 8-62\%) for patients with no detectable HLA class I allelic mismatch, low-resolution match/high-resolution mismatch, and low-resolution mismatch donors. Whereas OS was $49 \%(95 \% \mathrm{CI}, 6-51 \%)$ in both allele matched and one-allele mismatched patient/donor pairs, it was only $25 \%$ $(95 \% \mathrm{CI}, 11-75 \%)$ in two and more allele mismatched pairs (Figure 2). Thus, multiple allelic mismatches of HLA class I significantly $(P=0.03)$ decreased survival. In this patient group, causes of death were TRM in two-thirds of patients and relapse in one-third, whereas half of deaths in patients with no detectable HLA class I allelic mismatch or oneallele mismatched donors were TRM and the other half relapse.

In multivariate analysis, HLA class I allelic disparity had no significant impact $(P=0.18)$ on OS whereas high-risk disease prior to HSCT had a significant $(P=0.001)$ negative impact on survival as shown in Table 3 .

The Kaplan-Meier probability of DFS at 3 years was $41 \%$ (95\% CI, 6-59\%), 47\% (95\% CI, $11-53 \%$ ), and $40 \%$ $(95 \%$ CI, $8-60 \%)$ for patients with HLA class I allelic match, low-resolution match/high-resolution mismatch,

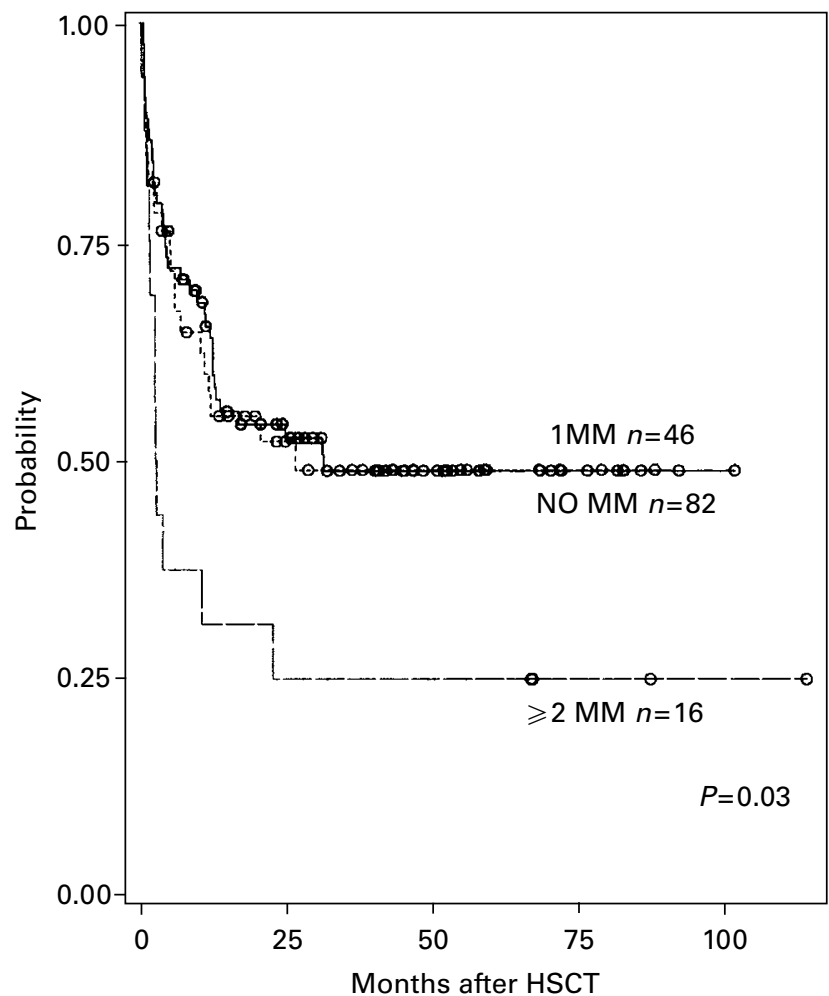

Figure 2 Impact of multiple HLA class I allelic disparities on OS. Probability of overall survival at 3 years is shown according to the number of HLA class I allele disparities between donor and recipient assessed by high-resolution typing. Groups comprise patients without allelic mismatches (thick solid line), with one allelic mismatch (including 29 with one antigen mismatch already detected by low-resolution typing, fine, broken line) and two or more allelic mismatches (including 16 with antigen mismatches already detected by low-resolution typing, thick broken line). 
and low-resolution mismatch donors. Only disease stage had a significant impact on DFS in univariate $(P \leqslant 0.0001)$ and multivariate analysis $(P=0.0001)$ as shown in Table 3 .

\section{Discussion}

For patients without an HLA-identical sibling, transplantation with hematopoietic stem cells from an unrelated donor may be an alternative. Unfortunately, the morbidity and mortality associated with such transplants are usually higher. Matching of HLA-A, B, C, and DRB1 led to reduction of risk of acute GVHD and improvement of survival after transplantation with URD. ${ }^{4,6,8,12,16-18}$ So far, few data on the impact of HLA class I and class II disparities on incidence and severity of chronic GVHD have been published. ${ }^{17,18}$ In a multicentre study reported by Morishima et $a l,{ }^{17}$ HLA-A/B allele disparities were significantly correlated with chronic GVHD by both univariate and multivariate analysis and HLA-C mismatch had a tendency to increase the incidence of chronic GVHD. We confirm these findings in a smaller but with regards to conditioning and GVHD prophylaxis more homogenous patient population. In addition, all our patients were matched for HLA-DRB1, DRB3/4/5, and DQB1 alleles. In our study, a significantly increased incidence of chronic GVHD was seen in patients with HLA class I allelic and antigen level mismatched unrelated donors. Furthermore, a single HLA class I allele mismatch significantly increased the risk of chronic GVHD at 3 years after HSCT from 21 to $44 \%$. In both univariate and multivariate analysis, only HLA class I disparity significantly increased the risk of chronic GVHD. Besides HLA disparity, age of recipient, type of GVHD prophylaxis and its duration, cytomegalovirus seropositivity, and prior acute GVHD are known risk factors for chronic GVHD. ${ }^{25}$ Whereas Morishima et $a l^{17}$ observed an effect of age on incidence of chronic GVHD, this was not seen in our study. However, the median age of their patient population was 23 years, 14 years less than ours. Furthermore, the power of the analysis may be compromised by the smaller number of patients in our study. Recently, Flomenberg et $a l^{18}$ reported a significantly adverse effect of HLA-A mismatches on chronic GVHD whereby these were more evident in transplants with low-resolution $v s$ only high-resolution mismatches.

In our study, survival probabilities were not significantly different in patients with no detectable HLA class I allelic mismatch donor and low-resolution match/high-resolution mismatched donors. We could confirm data published by Petersdorf et $a l^{7}$ where single class I disparities were well tolerated, whereas a significantly lower survival was observed when more than one class I allelic mismatch was present. While no statistically significant difference in incidence of severe acute GVHD was observed in the later group, there was a trend towards increased chronic extensive GVHD and TRM. Similarly, Flomenberg et al ${ }^{18}$ reported a progressively reduced survival with increased mismatches for HLA-A, B, and C.

Owing to small patient/donor pair numbers with HLA class I mismatches, no further analyses on the impact of various class I alleles on survival were performed in our study. Morishima et $a l^{17}$ observed a reduced OS due to worse nonrelapse mortality in patients given HLA-A and/ or HLA-B allele mismatch grafts, whereas the HLA-C mismatch or HLA class II (DRB1 and/or DQB1) mismatch did not. The favorable survival rate in single HLA-C mismatch graft recipients was mainly influenced by the low incidence of nonrelapse mortality, although relatively high incidence of severe GVHD occurred in HLA-C mismatch graft recipients compared with HLA matched ones. In contrast, Flomenberg et $a l^{18}$ demonstrated significant adverse impact for HLA-A, B, or C mismatching on survival. There, transplants with low-resolution mismatches were associated with significantly worse survival than those with only high-resolution mismatches.

In our study, $73 \%$ of donor/patient pairs were matched for HLA-A, B, C antigens and DRB1. Based on previous findings ${ }^{6,17,26}$ that HLA-C exerts significant effects on graft failure and survival, these HLA loci were included in our donor search algorithm. Recent publications confirm these findings. ${ }^{18,27}$ High-resolution typing revealed mismatches not detected by low-resolution typing in $24 \%$ of donor/ patient pairs reducing the percentage of fully matched pairs to $57 \%$. In all, $80 \%$ of mismatches involved HLA-B or C and two-thirds of mismatches were observed as single allelic ones. Thus, our study population had fewer HLA disparities compared to other reports. ${ }^{18}$ Nevertheless, HLA class I allelic mismatch was significantly associated with an increased risk for chronic GVHD. In view of the marked reduction of quality of life of patients with longlasting chronic GVHD, their need of prolonged immunosuppressive therapy and the significant reduction in overall survival of our patients with more than one-allelic HLA class I mismatch donors, selection of unrelated donors for transplantation should be based on high-resolution class I typing for HLA-A, B, and C.

\section{References}

1 Thomas ED. History, current results, and research in marrow transplantation. Perspect Biol Med 1983; 38: 230-237.

2 Armitage JO. Bone marrow transplantation. $N$ Engl $J$ Med 1994; 330: 827-838.

3 Kernan NA, Bartsch G, Ash RC et al. Analysis of 462 transplantations from unrelated donors facilitated by the National Marrow Donor Program. N Engl J Med. 1993; 328: 593-602.

4 Hansen JA, Gooley TA, Martin PJ et al. Bone marrow transplants from unrelated donors for patients with chronic myeloid leukemia. $N$ Engl J Med 1998; 338: 962-968.

5 Madrigal JA, Scott I, Arguello R et al. Factors influencing the outcome of bone marrow transplants using unrelated donors. Immunol Rev 1997; 157: 153-166.

6 Sasazuki T, Juji T, Morishima Y et al. Effect of matching of class I HLA alleles on clinical outcome after transplantation of hematopoietic stem cells from an unrelated donor. $N$ Engl $J$ Med 1998; 339: 1177-1185.

7 Petersdorf EW, Gooley TA, Anasetti C et al. Optimizing outcome after unrelated marrow transplantation by comprehensive matching of HLA class I and II alleles in the donor and recipient. Blood 1998; 92: 3515-3520. 
8 Speiser DE, Tiercy JM, Rufer $\mathrm{N}$ et al. High resolution HLA matching associated with decreased mortality after unrelated bone marrow transplantation. Blood 1996; 87: 4455-4462.

9 McGlave P, Bartsch G, Anasetti C et al. Unrelated donor marrow transplantation therapy for chronic myelogenous leukemia: initial experience of the National Marrow Donor Program. Blood 1993; 81: 543-550.

10 Nademanee A, Schmidt GM, Parker P et al. The outcome of matched unrelated donor bone marrow transplantation in patients with hematological malignancies using molecular typing for donor selection and graft-versus-host disease prophylaxis regimen of cyclosporine, methotrexate, and prednisone. Blood 1995; 86: 1228-1234.

11 Sanatamaria P, Reinsmoen NL, Lindstrom AL et al. Frequent HLA class I and DP sequence mismatches in serologically (HLA-A, HLA-B, HLA-DR) and molecularly (HLA-DRB1, HLA-DQA1, HLA-DQB1) HLA-identical unrelated bone marrow transplant pairs. Blood 1994; 83: 280-287.

12 Petersdorf EW, Longton GM, Anasetti $\mathrm{C}$ et al. The significance of HLA-DRB1 matching on clinical outcome after HLA-A, B, DR identical unrelated donor marrow transplantation. Blood 1995; 86: 1606-1613.

13 Petersdorf EW, Longton GM, Anasetti C et al. Definition of HLA-DQ as a transplantation antigen. Proc Natl Acad Sci USA 1996; 93: 15358-15363.

14 al-Daccak R, Loiseau P, Rabian C et al. HLA-DR, DQ, and/ or DP genotypic mismatches between recipient-donor pairs in unrelated bone marrow transplantation and transplant clinical outcome. Transplantation 1990; 50: 960-964.

15 Varney MD, Lester S, McCluskey $\mathbf{J}$ et al. Matching for HLA DPA1 and DPB1 alleles in unrelated bone marrow transplantation. Hum Immunol 1999; 60: 532-538.

16 Petersdorf EW, Kollman C, Katovich Hurley C et al. Effect of HLA class II gene disparity on clinical outcome in unrelated donor hematopoietic cell transplantation for chronic myeloid leukemia: the US National Marrow Donor Program Experience. Blood 2001; 98: 2922-2929.

17 Morishima Y, Sasazuki T, Inoko $\mathrm{H}$ et al. The clinical significance of human leukocyte antigen (HLA) allele compat- ibility in patients receiving a marrow transplant from serologically HLA-A, HLA-B, and HLA-DR matched unrelated donors. Blood 2002; 99: 4200-4206.

18 Flomenberg N, Baxter-Lowe LA, Confer D et al. Impact of HLA class I and class Ii high resolution matching on outcomes of unrelated donor bone marrow transplantation: HLA-C mismatching is associated with a strong adverse effect on transplant outcome. Blood 2004; prepublished online June 10, 2004.

19 Rowlings PA, Przepiorka D, Klein JP et al. IBMTR severity index for grading acute graft-versus-host disease: Retrospective comparison with Glucksberg grade. $\mathrm{Br} J$ Haematol 1997; 97: 855-864.

20 Sullivan KM, Shulman HM, Storb R et al. Chronic graftversus-host disease in 52 patients: adverse natural course and successful treatment with combination immunosuppression. Blood 1981; 57: 267-276.

21 Mittal KK, Mickey MR, Singal DP, Terasaki PI. Serotyping for homotransplantation. Refinement of microdroplet lymphocyte cytotoxicity test. Transplantation 1968; 6: 913-927.

22 Fischer GF, Faé I, Petrasek M et al. A combination of two distinct in-vitro amplification procedures for DNA typing of HLA-DRB and -DQB1 alleles. Vox Sang 1995; 69: 328-335.

23 Fischer GF, Fae I, Frey E, Mayr WR. HLA-A*02172 adds to the heterogeneity of HLA-A*02 alleles. Tissue Antigens 1998; 51: $312-314$

24 Gray RJ. A class of K-sample tests for comparing the cumulative incidence of a competing risk. Ann Statist 1988; 16: 1141-1154.

25 Vogelsang GB. How I treat chronic graft-versus-host disease. Blood 2001; 97: 1196-1202.

26 Petersdorf EW, Longton GM, Anasetti C et al. Associations of HLA-C disparity with graft failure after marrow transplantation from unrelated donors. Blood 1997; 89: 1818-1823.

27 Tiercy JM, Passweg J, van Biezen A et al. Isolated HLA-C mismatches in unrelated donor transplantation for CML. Bone Marrow Transplant 2004; advanced online publication 7 June 2004. 